\title{
Sustained Cerebrovascular and Cognitive Benefits of Resveratrol in Postmenopausal Women
}

\author{
Jay Jay Thaung Zaw ${ }^{1}$, Peter R. C. Howe ${ }^{1,2,3}$ and Rachel H. X. Wong ${ }^{1,2, *(1)}$ \\ 1 School of Biomedical Sciences and Pharmacy, University of Newcastle, Callaghan 2308, New South Wales, \\ Australia; JayJay.ThaungZaw@uon.edu.au (J.J.T.Z.); peter.howe@newcastle.edu.au (P.R.C.H.) \\ 2 Institute for Resilient Regions, Springfield Central, University of Southern Queensland, \\ Springfield Central 4300, Queensland, Australia \\ 3 School of Health Sciences, University of South Australia, Adelaide 5000, South Australia, Australia \\ * Correspondence: Rachel.wong@newcastle.edu.au; Tel.: +61-2-4921-6408
}

Received: 14 February 2020; Accepted: 17 March 2020; Published: 20 March 2020

\begin{abstract}
Deficits in the cerebral microcirculation contribute to age-related cognitive decline. In a pilot study of postmenopausal women, we found that supplementation with a low dose of resveratrol, a phytoestrogen, for 14 weeks improved cerebrovascular and cognitive functions. We have since undertaken a larger, longer term study to confirm these benefits. Postmenopausal women aged $45-85$ years $(n=129)$ were randomized to take placebo or $75 \mathrm{mg}$ trans-resveratrol twice daily for 12 months. Effects on cognition, cerebral blood flow, cerebrovascular responsiveness (CVR) and cardiometabolic markers (blood pressure, diabetes markers and fasting lipids) were assessed. Compared to placebo, resveratrol improved overall cognitive performance $(P<0.001)$ and attenuated the decline in CVR to cognitive stimuli $(P=0.038)$. The latter effect was associated with reduction of fasting blood glucose $(\mathrm{r}=-0.339, P=0.023)$. This long-term study confirms that regular consumption of resveratrol can enhance cognitive and cerebrovascular functions in postmenopausal women, with the potential to slow cognitive decline due to ageing and menopause.
\end{abstract}

Keywords: resveratrol; ageing; menopause; cognitive decline; cerebrovascular function; neurovascular coupling; phytoestrogen; nutraceutical

\section{Background}

Since 1990, the number of people globally living with dementia has more than doubled. This is mainly due to increased population growth and ageing [1]. Importantly, independent of life expectancy, dementia mortality rates in women in 2016 were almost twice that of men. This may be partly attributable to the abrupt decline of estrogen at menopause and the associated loss of its protective effects on cardiovascular [2] and neural functions [3].

Estrogen activates estrogen $\alpha$ and $\beta$ receptors (ER) on endothelial cells to facilitate vasodilatation by increasing endothelial nitric oxide (NO). Thus, estrogen deprivation can accelerate age-related arterial stiffening and impair tissue perfusion by reducing endothelium-dependent vasodilatation. This not only increases the risk of cardiovascular disease postmenopausally [4] but also reduces cerebrovascular responsiveness (CVR) in postmenopausal women compared to pre-menopausal women and men $[5,6]$. Reduced cerebral blood flow $(\mathrm{CBF})$ and CVR are associated with cognitive impairment [7]. We have also reported that reduced CVR during mental task activation (neurovascular coupling) is predictive of poor cognitive performance in postmenopausal women [8]. A meta-analysis has shown that postmenopausal women perform worse on verbal memory and executive function tests compared to peri-menopausal women [9]. Therefore, maintaining the health of the cerebral vasculature may slow cognitive decline in postmenopausal women. 
Resveratrol (3,5,4'-trihydroxy-trans-stilbene), a phytoestrogen present in a variety of foods such as grapes, berries and nuts, has been shown to improve endothelial vasodilator function in humans [10]. Resveratrol can act through multiple mechanisms including activation of endothelial ER to increase NO production and thereby facilitate endothelium-dependent vasodilatation necessary for adequate cerebral perfusion [11]. Resveratrol has been shown to improve verbal memory in older adults after six months of $200 \mathrm{mg}$ of supplementation per day compared to placebo [12]. Using functional magnetic resonance imaging, Witte et al. found that resting-state functional connectivity in the hippocampus (a region critical for memory functions) and other brain regions was significantly increased in the resveratrol group and was correlated with improvement in verbal memory [12]. Kennedy et al. reported that compared to placebo, acute doses of $250 \mathrm{mg}$ and $500 \mathrm{mg}$ of resveratrol resulted in dose-dependent increases of resting CBF in younger adults, although cognitive function was not affected [13]. In a dose-response evaluation, Wong et al. found that $75 \mathrm{mg}$ resveratrol, the lowest dose tested, was the most efficacious dose to acutely elicit global cerebral vasodilatation [14] and improve performance of a sustained attention task in type 2 diabetes patients [15]. In a subsequent pilot study of chronic resveratrol treatment $(2 \times 75 \mathrm{mg} /$ day for 14-weeks) in 80 postmenopausal women, we observed an improvement of cognitive performance, which was accompanied by enhanced neurovascular coupling, suggesting that regular resveratrol supplementation might attenuate accelerated cognitive ageing in a vulnerable population by helping to maintain normal circulatory function [16]. We now intend to investigate whether these benefits can be sustained long-term as well as examining its effects on a wide range of cardiometabolic markers.

\section{Subjects and Methods}

\subsection{Study Design}

A 24-month randomised, double-blind, placebo-controlled (crossover comparison) dietary intervention trial was conducted to evaluate the effects of resveratrol supplementation (75 mg twice daily) on cognitive performance to a neuropsychological test battery and on associated cerebrovascular and cardiometabolic markers in post-menopausal women. This manuscript will report an interim analysis performed at the end of first stage of crossover to determine the within-individual treatment changes (post-pre supplementation) between the placebo and resveratrol arm. For $90 \%$ power to detect a statistically significant $(P<0.05)$ medium effect size (Cohen's $d=0.5$ ) improvement in the primary outcome (overall cognitive performance), 87 completers were required for a crossover comparison. To allow for $45 \%$ attrition due to a long-term study and to account for the difficulty in detecting an acoustic temporal window for cerebrovascular function assessments, especially in elderly women, we aimed to recruit 170 women. The trial was conducted at the Clinical Nutrition Research Centre of the University of Newcastle in New South Wales, Australia in accordance with the Declaration of Helsinki and the Principles of Good Clinical Practice as outlined by the International Conference on Harmonisation. The protocol was approved by the University of Newcastle's Human Research Ethics Committee (H-2016-0091) and registered with the Australian and New Zealand Clinical Trial Registry (ACTRN12616000679482p).

\subsection{Study Population}

From November 2016 to May 2017, community-dwelling postmenopausal women residing in the Hunter region of New South Wales were recruited through approved newspaper and radio campaigns and from a database of previous Clinical Nutrition Research Centre participants and from the Hunter Medical Research Institute Volunteer Registry. Interested volunteers were provided with a detailed information sheet and completed a health and lifestyle questionnaire to determine whether they met the inclusion criteria of the study. Eligible participants were aged 45-85 years, $>12$ months post-menopausal and willing to maintain their current lifestyle throughout the study. We did not include volunteers if they took hormone replacement therapy, insulin or warfarin within the past six 
months, had suspected dementia or clinical depression. To avoid possible drug interactions, hormonal influences and other pathological brain lesions causing cognitive impairments, individuals who had a history of breast or cervical cancer, major cardiovascular, kidney or liver disease or a neurological disorder were also excluded.

\subsection{Screening Visit and Follow-Up Assessments}

Potentially eligible participants attended the screening/baseline visit with at least two hours fasting (no medication, food or caffeinated or sugary beverages). Written informed consent was obtained prior to any assessments. A clinical investigator measured height $(\mathrm{m})$, weight $(\mathrm{kg})$, and waist circumference at the narrowest part of midriff $(\mathrm{cm})$. Body mass index (BMI) was derived by dividing weight by height ${ }^{2}$. Seated blood pressure (BP) was assessed after 10 minutes of rest and those above 160/100 mmHg were excluded. Participants' global cognitive status was assessed using the Australian Version of Addenbrooke's Cognitive Examination III (ACE-III), where the cut-off for suspected dementia is less than $82 \%$ [17]. Those who met full eligibility criteria were enrolled and invited for follow-up assessments. Participants were instructed to refrain from consuming their supplement on the day of any visit to assess the sustained effects of resveratrol.

\subsection{Investigational Product and Allocation}

Resveratrol (Veri-te ${ }^{\mathrm{TM}}$ ) and placebo capsules were identical in shape and colour and supplied by Evolva SA, Switzerland. Each resveratrol capsule contained $75 \mathrm{mg}$ of $>98 \%$ pure synthetic trans-resveratrol and placebo comprised of several inert excipients. Containers of capsules were only identifiable by code numbers; an independent investigator who held the code allocated volunteers to resveratrol or placebo capsules using Altman's randomization by minimisation procedure [18] in order to balance treatment groups based on age, postmenopausal years and clinic blood pressure (measured at the screening visit).

\subsection{Outcome Assessments}

\subsubsection{Clinic Blood Pressure and Arterial Compliance}

Seated BP was taken with an appropriately sized cuff placed over the brachial artery of the non-dominant hand (Cardiovascular Profiler CR 2000, Minnesota, MN, USA). The dominant hand was stabilized by a rigid wrist support and a tonometer was attached perpendicularly over the radial artery to measure the arterial compliance (AC) of systemic arteries. Three repeated measures of BP and AC were performed at two-minute intervals. The first BP measurement was discarded and the remaining readings were averaged.

\subsubsection{Cerebrovascular Function Assessments with Transcranial Doppler Ultrasound}

\section{Basal Cerebral Haemodynamics}

A transcranial Doppler ultrasound (TCD) headpiece (Doppler-Box X, Singen, Germany) was fitted on participants' heads with probes on the left and right temporal area to insonate middle cerebral arteries (MCA) at a depth of 45-60 mm. TCD is a non-invasive technique to assess the changes in blood flow velocities (BFV) in the brain [19]. A 30-second continuous recording of basal BFV (maximum, minimum, mean) was obtained before hypercapnic provocation and before the start of each cognitive test. Stiffness in the cerebral vessels or pulsatility index (PI) at baseline was derived as follows: (maximum BFV - minimum BFV)/mean BFV.

Cerebrovascular Responsiveness (CVR)

CVR to hypercapnia: Participants breathed in carbogen gas $\left(95 \% \mathrm{O}_{2}, 5 \% \mathrm{CO}_{2}\right)$ through a two-way non-rebreathing mouthpiece for 180 seconds. TCD recorded the increases in bilateral beat-to-beat 
mean BFV throughout the hypercapnic challenge. A further 60 seconds recording was taken whilst the participants inhaled normal room air to ensure that their BFV returned to resting values.

CVR to cognitive stimuli (neurovascular coupling): The TCD was kept in position throughout the neuropsychological test battery to assess CVR to cognitive stimuli, and it was recorded before (30-second baseline) and during each cognitive task.

CVR recordings were smoothed and analysed in TableCurve ${ }^{\mathrm{TM}}$ (TableCurve 2D by Systat Software Inc., San Jose, CA, USA) using data spline estimation with Loess at $20 \%$ for hypercapnia assessments and $10 \%$ for neurovascular coupling to determine the peak increase in mean BFV. CVR was calculated as follows: [(peak mean BFV - resting mean BFV)/resting mean BFV $\times 100]$.

\subsubsection{Cognitive Performance}

The cognitive test battery consisted of seven cognitive tests from the National Institutes of Health Toolbox (NIH-ToolBox) assessment [20] and three other validated cognitive tests viz. Rey's Auditory Verbal Learning Test (RAVLT), Forward Spatial Span Test and Trail Making Task. Relevant tests chosen for cognitive domains were depicted in Table 1 . This battery provided different versions of the tests to administer at baseline and after 12 months, thus avoiding practice effects.

A trained study investigator delivered the test battery (except for the Trail Making Task) on an iPad, which took about 90 minutes to complete. Data were stored in the device, transferred securely and backed-up every week. Practice runs preceded each test to ensure the participants' full understanding of the instructions.

Table 1. Cognitive domains and component tasks in the neuropsychological test battery.

\begin{tabular}{ccc}
\hline Cognitive Domains & NIH-ToolBox Assessment & Other Assessment \\
\hline Processing speed & Pattern Comparison Speed Test & Trail Making Task A \\
\hline Language & $\begin{array}{c}\text { Picture Vocabulary Test } \\
\text { Oral Reading Recognition Test }\end{array}$ & \\
\hline Working memory & List Sorting Working Memory Test & Forward Spatial Span test \\
\hline Episodic memory & Picture Sequence Memory Test & $\begin{array}{c}\text { Rey's Auditory Verbal Learning } \\
\text { Test (immediate recall and } \\
\text { 30-minute delayed recall) }\end{array}$ \\
\hline Verbal memory & & Trail Making Task B \\
\hline Cognitive flexibility & $\begin{array}{c}\text { Dimensional Change Card Sort Test } \\
\text { Flanker Inhibitory Control and } \\
\text { Attention Test }\end{array}$ & \\
\hline
\end{tabular}

\subsubsection{Blood Biomarker Assessment}

Overnight fasted venous blood samples were collected by a phlebotomist at baseline and after 12 months. The biomarker analysis included fasting serum glucose, insulin, lipids (total cholesterol, triglycerides, HDL and LDL-cholesterol) and high-sensitivity C-reactive protein (hs-CRP) as a marker of systemic inflammation. Homeostatic Model Assessment of Insulin Resistance (HOMA-IR) was derived from the fasting glucose and insulin results.

\subsection{Intervention}

Participants were instructed to take two capsules of their allocated treatment each day (one in the morning and one in the evening). A supplement diary was provided to record the time that the capsules were taken and any changes to medications or habitual lifestyle. If a dose was missed, participants were allowed to catch up on the same day but not to double-up the next day. There was a face-to-face compliance check at month-6 where the participants were required to attend an hour-long clinic visit to undergo BP and CVR assessments and acquire new supplement bottles and diaries. 
All unused capsules were returned at the six- and 12-month visits and were counted and tallied with supplement diaries to monitor compliance. In addition, a study investigator made phone calls every three months to track participants' well-being, lifestyle or medication changes and any occurrence of illness or side effects.

\subsection{Statistical Analysis}

Treatments by time effects were determined by analysis of variance (ANOVA) using SPSS version 25.0 (SPSS by IBM Inc. Chicago, IL, USA). The primary outcome was the treatment change from baseline in cognitive performance, determined using a composite score (z-scores) of the neuropsychological test battery between resveratrol and placebo groups. The scores from each cognitive task obtained at 12 months were converted to z-scores derived from the cohort's performance at baseline. Effect sizes were calculated using Cohen's $d$ [21]. Secondary outcomes were the treatment by time effects on BFV, PI, CVR to hypercapnia, CVR to cognitive stimuli and cardiometabolic markers. Pearson's correlational analysis was applied to examine the associations between cerebrovascular function and other outcome measures. The Benjamini-Hochberg procedure [22] was used to correct P-values for secondary outcomes (cerebrovascular function and cardiometabolic markers) to minimise type I errors; the false discovery rate was set at 0.15 . Data are presented as mean \pm SEM (standard error of the mean) unless otherwise stated.

\section{Results}

\subsection{Participant Disposition}

Figure 1 depicts the participant disposition of this study in accordance with CONSORT (Consolidated Standards of Reporting Trials) statement 2010 [23]. Although we formerly aimed to recruit 170 participants, due to logistical limitations, we invited 151 women for a screening visit and enrolled 146 participants, of which 129 participants completed the 12-month trial and were included in the analysis of the primary outcome. This provided $80 \%$ power at alpha 0.05 , to detect a statistically significant medium effective size improvement (Cohen's $d=0.5$ ) in the primary outcome for a parallel comparison. Of 17 participants (12\%) who withdrew their participation before 12 months, 10 had been allocated resveratrol and seven placebo.

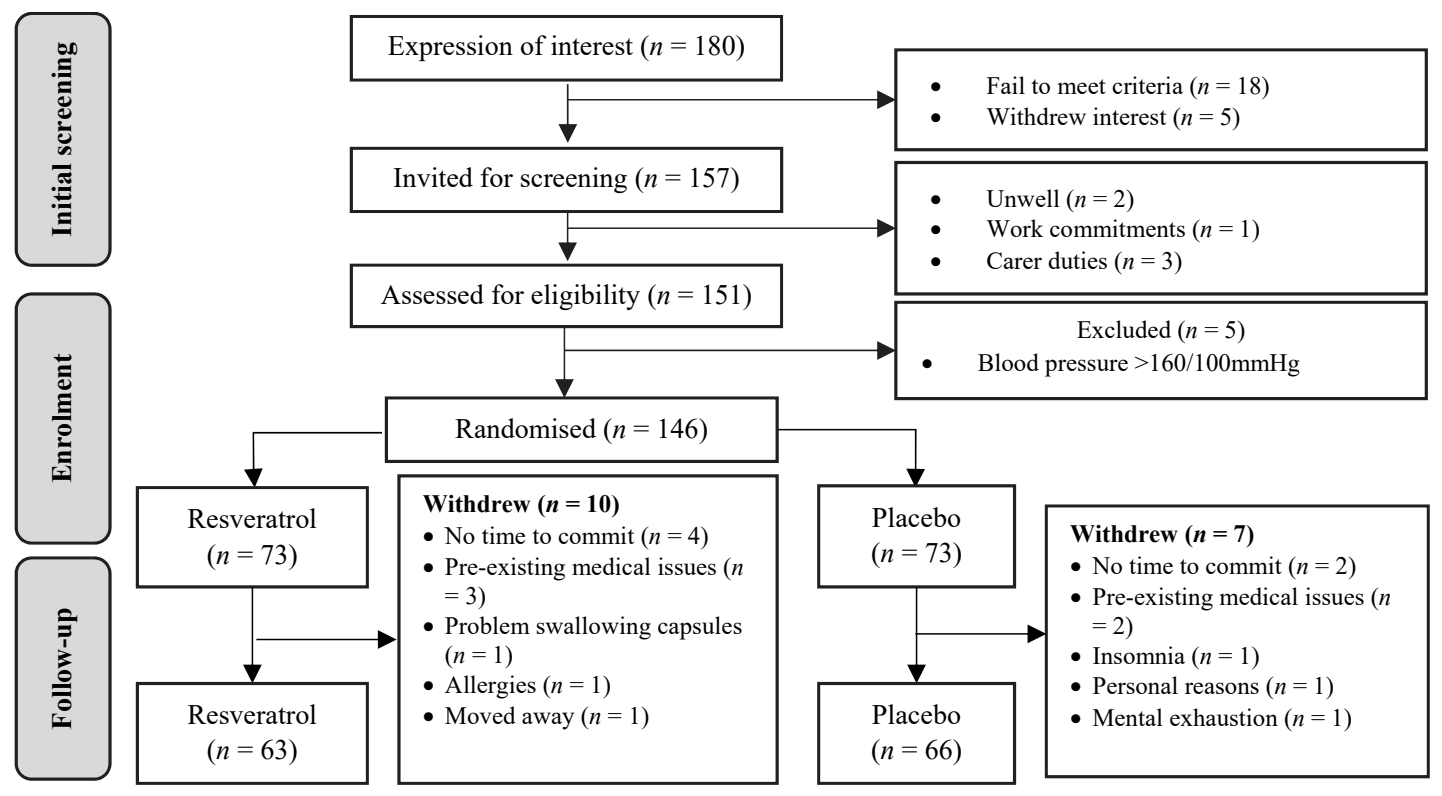

Figure 1. CONSORT diagram. Flow of participants from initial contact until final assessment. 


\subsection{Baseline Characteristics}

Table 2 shows baseline characteristics of 146 women who were enrolled and randomized to placebo $(n=73)$ and resveratrol $(n=73)$ groups. Averaging 64 years of age and 15 years post-menopausal, they were slightly overweight, normotensive, well-educated and cognitively unimpaired, as indicated by ACE-III scores. There were no differences between groups.

Table 2. Participant baseline characteristics $(n=146)$.

\begin{tabular}{cccc}
\hline Participant's Characteristics & Total $(\boldsymbol{n = 1 4 6 )}$ & Placebo $(\boldsymbol{n = 7 3 )}$ & Resveratrol $(\boldsymbol{n}=\mathbf{7 3})$ \\
\hline Age (years) & $64 \pm 1$ & $64 \pm 1$ & $64 \pm 1$ \\
\hline Years since cessation of menses & $15 \pm 1$ & $15 \pm 1$ & $15 \pm 1$ \\
\hline Years of education & $17 \pm 0.3$ & $17 \pm 0.5$ & $17 \pm 0.5$ \\
\hline ACE-III score $(\%)$ & $93 \pm 0.5$ & $93 \pm 0.7$ & $93 \pm 0.5$ \\
\hline BMI $\left(\mathrm{kg} / \mathrm{m}^{2}\right)$ & $25.6 \pm 0.3$ & $25.8 \pm 0.5$ & $25.4 \pm 0.5$ \\
\hline Systolic blood pressure $(\mathrm{mmHg})$ & $124 \pm 1$ & $125 \pm 2$ & $123 \pm 2$ \\
\hline Diastolic blood pressure $(\mathrm{mmHg})$ & $68 \pm 1$ & $69 \pm 1$ & $67 \pm 1$ \\
\hline Large artery compliance $(\mathrm{mL} / \mathrm{mmHg} \times 10)$ & $12.6 \pm 0.36$ & $12.1 \pm 0.38$ & $13.1 \pm 0.67$ \\
\hline Small artery compliance $(\mathrm{mL} / \mathrm{mmHg} \times 100)$ & $3.7 \pm 0.17$ & $3.7 \pm 0.29$ & $3.5 \pm 0.18$ \\
\hline
\end{tabular}

\subsection{Systemic Vascular Function}

Blood pressure and systemic arterial compliance (elasticity of both large and small arteries) remained unchanged after 12 months supplementation in both treatment groups (see Supplementary Materials Table S1).

\subsection{Cognitive Performance}

Performance of the Pattern Comparison Speed test improved with resveratrol supplementation compared to placebo. There were no other significant changes in individual tests (Table 3). However, improvements were seen in two cognitive domains, viz. processing speed $(P=0.019$, Cohen's $d=0.25)$ and cognitive flexibility $(P=0.011$, Cohen's $d=0.30)$, resulting in a modest increase in overall cognitive performance $(P=<0.001$, Cohen's $d=0.18$ ) (Figure 2 ).

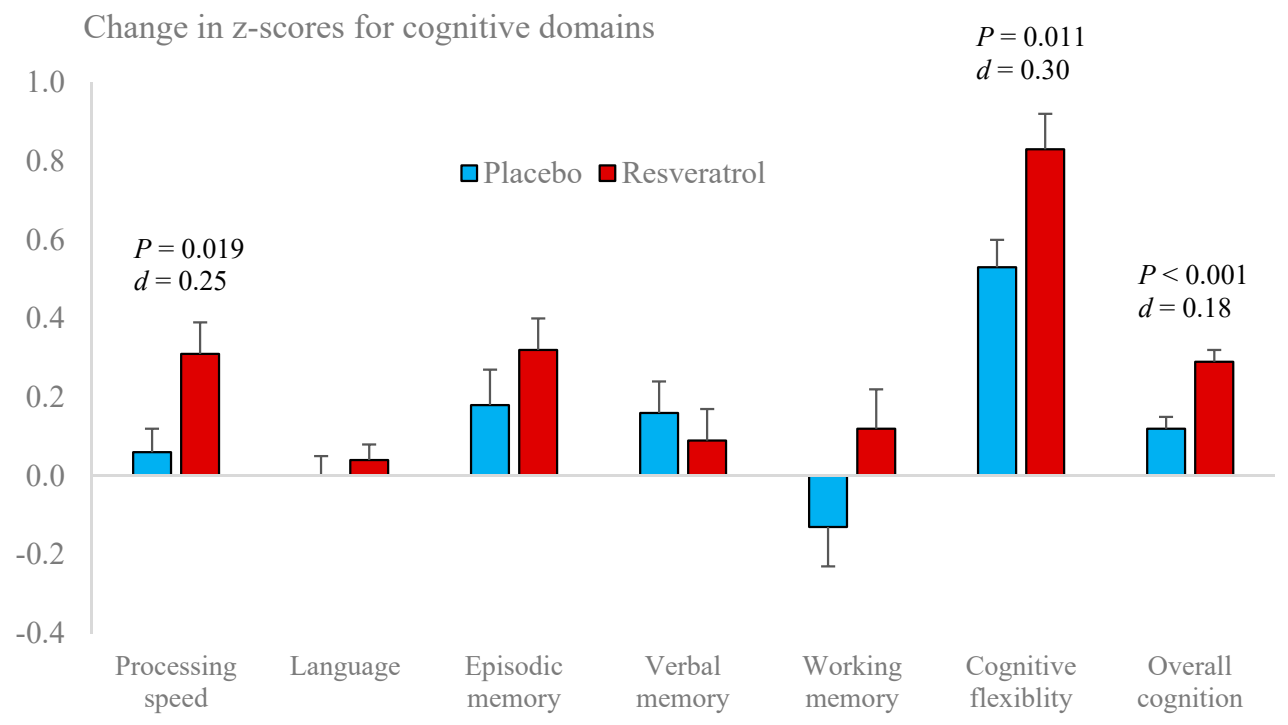

Figure 2. Performance changes in cognitive domains following placebo and resveratrol treatments. 
Table 3. Participants' z-scores for each cognitive domain and individual cognitive tests between resveratrol and placebo groups following 12 months supplementation.

\begin{tabular}{lcccc}
\hline \multirow{2}{*}{$\begin{array}{c}\text { Cognitive Domains } \\
\bullet \text { Component Tasks }\end{array}$} & $\begin{array}{c}\text { Placebo } \\
(\boldsymbol{n}=\mathbf{7 3})\end{array}$ & $\begin{array}{c}\text { Resveratrol } \\
(\boldsymbol{n}=\mathbf{7 3})\end{array}$ & $\begin{array}{c}\text { Placebo } \\
(\boldsymbol{n}=\mathbf{6 6})\end{array}$ & $\begin{array}{c}\text { Resveratrol } \\
(\boldsymbol{n}=\mathbf{6 3})\end{array}$ \\
\hline Processing Speed & $0.05 \pm 0.09$ & $-0.08 \pm 0.10$ & $0.23 \pm 0.10$ & $0.24 \pm 0.09 *$ \\
$\bullet$ PCT & $0.08 \pm 0.12$ & $-0.13 \pm 0.13$ & $0.15 \pm 0.13$ & $0.32 \pm 0.11^{*}$ \\
$\bullet$ TMT A & $0.02 \pm 0.13$ & $-0.02 \pm 0.11$ & $0.31 \pm 0.11$ & $0.16 \pm 0.11$ \\
\hline Language & $-0.03 \pm 0.10$ & $0.03 \pm 0.11$ & $0.04 \pm 0.11$ & $0.09 \pm 0.11$ \\
$\bullet$ PVT & $-0.09 \pm 0.12$ & $0.09 \pm 0.12$ & $0.05 \pm 0.13$ & $0.20 \pm 0.13$ \\
$\bullet$ ORR & $0.02 \pm 0.12$ & $-0.02 \pm 0.12$ & $0.04 \pm 0.10$ & $-0.02 \pm 0.11$ \\
\hline Episodic Memory & $-0.49 \pm 0.09$ & $-0.47 \pm 0.10$ & $-0.27 \pm 0.10$ & $-0.19 \pm 0.11$ \\
$\bullet$ PSM & $-0.49 \pm 0.09$ & $-0.47 \pm 0.10$ & $-0.27 \pm 0.10$ & $-0.19 \pm 0.11$ \\
\hline Verbal Memory & $0.001 \pm 0.1$ & $-0.001 \pm 0.1$ & $0.17 \pm 0.12$ & $0.12 \pm 0.10$ \\
$\bullet$ RAVLT immediate & $0.02 \pm 0.07$ & $-0.02 \pm 0.07$ & $0.07 \pm 0.06$ & $0.07 \pm 0.06$ \\
$\bullet$ RAVLT delayed & $-0.02 \pm 0.15$ & $0.02 \pm 0.15$ & $0.28 \pm 0.19$ & $0.17 \pm 0.16$ \\
\hline Working Memory & $0.04 \pm 0.10$ & $-0.04 \pm 0.10$ & $0.06 \pm 0.09$ & $0.05 \pm 0.09$ \\
$\bullet$ LSWM & $0.11 \pm 0.11$ & $-0.11 \pm 0.12$ & $0.08 \pm 0.14$ & $-0.03 \pm 0.13$ \\
$\bullet$ FSS & $-0.04 \pm 0.12$ & $0.04 \pm 0.11$ & $0.06 \pm 0.12$ & $0.12 \pm 0.10$ \\
\hline Cognitive Flexibility & $-0.03 \pm 0.12$ & $0.03 \pm 0.08$ & $0.78 \pm 0.08$ & $0.82 \pm 0.07 *$ \\
$\bullet$ DCCS & $-0.05 \pm 0.14$ & $0.05 \pm 0.09$ & $0.10 \pm 0.13$ & $0.23 \pm 0.10$ \\
$\bullet$ FICA & $-0.08 \pm 0.12$ & $0.08 \pm 0.11$ & $0.22 \pm 0.11$ & $0.26 \pm 0.12$ \\
$\bullet$ TMT performance & $0.02 \pm 0.21$ & $-0.02 \pm 0.19$ & $2.01 \pm 0.10$ & $1.97 \pm 0.11$ \\
\hline Overall Cognitive Performance & $-0.08 \pm 0.07$ & $-0.09 \pm 0.05$ & $0.17 \pm 0.06$ & $0.18 \pm 0.05 *$
\end{tabular}

NOTE. * Significant treatment change between resveratrol and placebo, Analysis of Variance, $P<0.05$. Abbreviations: DCCS $=$ Dimensional Change Card Sort, PVT = Picture Vocabulary, FICA = Flanker Inhibitory and Control Attention, PSM = Picture Sequence Memory, PCT = Pattern Comparison Speed, LSWM = List Sorting Working Memory, ORR $=$ oral reading recognition, RAVLT $=$ Rey's Auditory Verbal Learning Test, FSS $=$ Forward Spatial Span, $\mathrm{TMT}=$ Trail Making Task.

Improvements of overall cognitive performance in the resveratrol-treated group were greater in those individuals with lower cognitive performance at baseline $(\mathrm{R}=-0.380, P=0.002)$.

\subsection{Cerebrovascular Function}

Basal BFV (systolic, mean and diastolic) and PI were significantly improved with resveratrol (Figure 3). CVR to hypercapnia was unaffected by resveratrol but a decline in neurovascular coupling capacity was attenuated by resveratrol (Table 4). Overall neurovascular coupling capacity, especially in response to tests of cognitive flexibility, was improved (Figure 4).

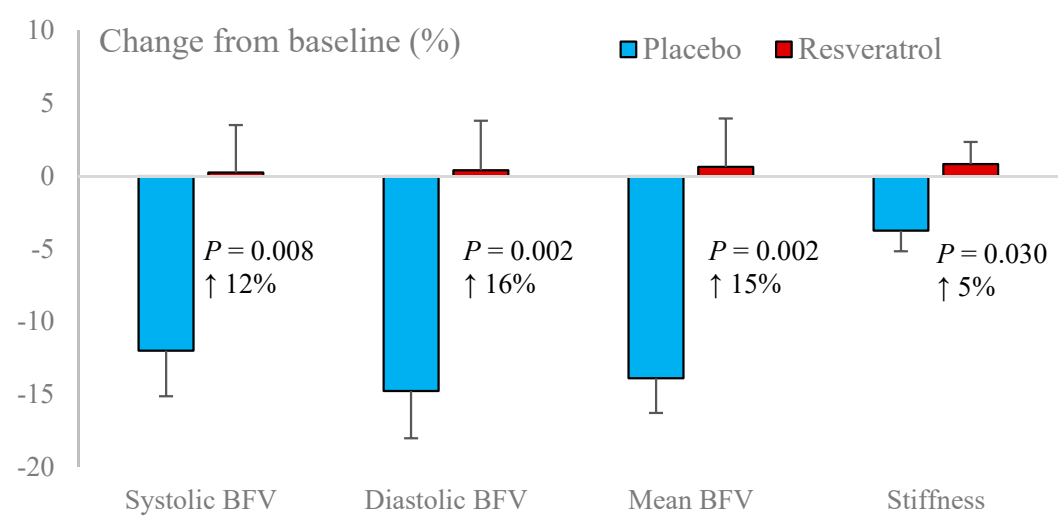

Figure 3. Changes in basal blood flow velocity (BFV) and pulsatility index (arterial stiffness) following resveratrol and placebo treatments. 
Table 4. Indices of cerebrovascular function between resveratrol and placebo groups at baseline and after 12 months of supplementation. Data are presented as mean \pm SEM.

\begin{tabular}{|c|c|c|c|c|}
\hline \multirow{2}{*}{$\begin{array}{c}\text { Cerebral Hemodynamics } \\
\text { Resting conditions }\end{array}$} & \multicolumn{2}{|c|}{ Month 0} & \multicolumn{2}{|c|}{ Month 12} \\
\hline & $\begin{array}{l}\text { Placebo } \\
(n=51)\end{array}$ & $\begin{array}{l}\text { Resveratrol } \\
\quad(n=47)\end{array}$ & $\begin{array}{l}\text { Placebo } \\
(n=51)\end{array}$ & $\begin{array}{c}\text { Resveratrol } \\
(n=47)\end{array}$ \\
\hline Systolic blood flow velocity $(\mathrm{cm} / \mathrm{s})$ & $81.3 \pm 2.7$ & $76.0 \pm 2.9$ & $71.8 \pm 1.9$ & $76.2 \pm 3.3$ \\
\hline Diastolic blood flow velocity $(\mathrm{cm} / \mathrm{s})$ & $36.0 \pm 1.4$ & $32.7 \pm 1.3$ & $30.9 \pm 0.95$ & $32.8 \pm 1.4$ \\
\hline Mean blood flow velocity (cm/s) & $53.8 \pm 1.9$ & $49.6 \pm 1.9$ & $46.6 \pm 1.3$ & $49.9 \pm 2.1$ \\
\hline Pulsatility index & $0.84 \pm 0.02$ & $0.87 \pm 0.03$ & $0.88 \pm 0.02$ & $0.86 \pm 0.02$ \\
\hline \multirow{2}{*}{$\begin{array}{c}\text { Cerebrovascular responses to } \\
\text { hypercapnia }(\%)\end{array}$} & $\begin{array}{l}\text { Placebo } \\
(n=44)\end{array}$ & $\begin{array}{l}\text { Resveratrol } \\
\quad(n=41)\end{array}$ & $\begin{array}{l}\text { Placebo } \\
(n=44)\end{array}$ & $\begin{array}{c}\text { Resveratrol } \\
(n=41)\end{array}$ \\
\hline & $45.8 \pm 1.7$ & $46.4 \pm 2.3$ & $47.2 \pm 2.03$ & $48.9 \pm 2.05$ \\
\hline Neurovascular coupling capacity $(\%)$ & $\begin{array}{l}\text { Placebo } \\
(n=45)\end{array}$ & $\begin{array}{l}\text { Resveratrol } \\
\quad(n=46)\end{array}$ & $\begin{array}{l}\text { Placebo } \\
(n=45)\end{array}$ & $\begin{array}{c}\text { Resveratrol } \\
(n=46)\end{array}$ \\
\hline Processing speed & $19.2 \pm 1.4$ & $19.6 \pm 1.4$ & $15.4 \pm 1.3$ & $16.9 \pm 1.2$ \\
\hline Language & $14.0 \pm 1.1$ & $12.0 \pm 0.97$ & $11.9 \pm 0.95$ & $11.6 \pm 0.63$ \\
\hline Episodic memory & $16.6 \pm 1.3$ & $14.5 \pm 1.2$ & $13.5 \pm 1.07$ & $14.2 \pm 1.2$ \\
\hline Verbal memory & $15.9 \pm 1.2$ & $16.2 \pm 1.02$ & $12.6 \pm 0.89$ & $12.4 \pm 0.85$ \\
\hline Working memory & $15.5 \pm 1.02$ & $14.2 \pm 0.94$ & $13.4 \pm 0.83$ & $13.0 \pm 0.64$ \\
\hline Cognitive flexibility & $16.1 \pm 0.94$ & $14.3 \pm 0.92$ & $11.7 \pm 0.77$ & $13.5 \pm 0.74$ \\
\hline Overall cognition & $16.4 \pm 0.73$ & $15.0 \pm 0.63$ & $12.8 \pm 0.69$ & $13.5 \pm 0.59$ \\
\hline
\end{tabular}

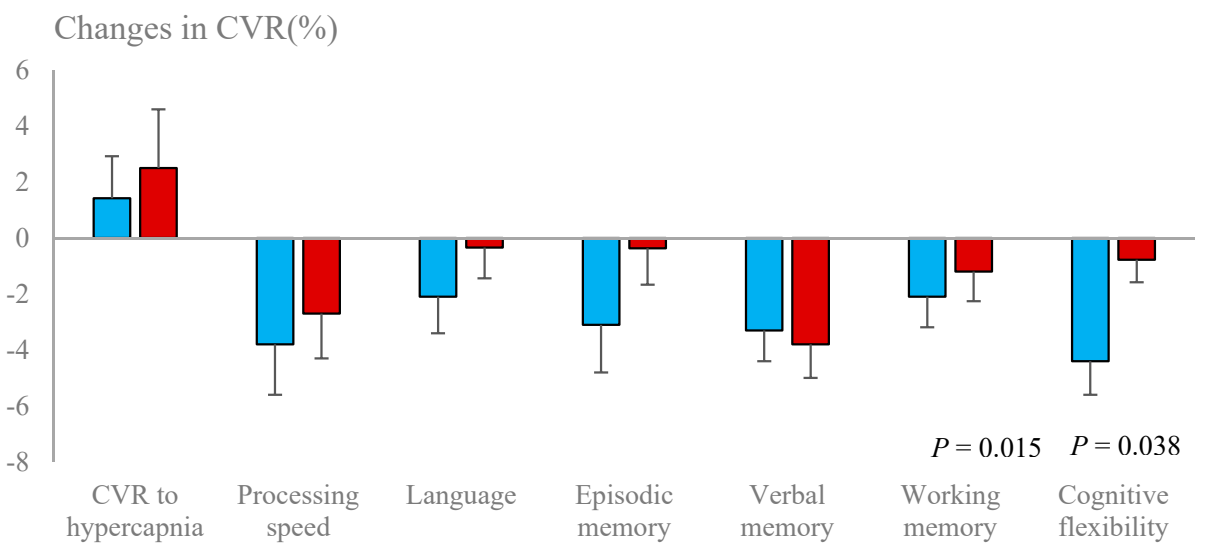

$\square$ Placebo $\square$ Resveratrol

Figure 4. Changes in cerebrovascular responsiveness (CVR) to hypercapnia and to cognitive tests following resveratrol and placebo treatments.

\subsection{Cardiometabolic Markers}

There were no significant differences in cardiometabolic markers between placebo and resveratrol groups (Table 5). However, we observed that reductions in fasting blood glucose were associated with the improvements in overall neurovascular coupling capacity following resveratrol supplementation $(\mathrm{r}=-0.339, P=0.023)$ (Figure 5). 
Table 5. Cardiometabolic markers between resveratrol and placebo groups at baseline and after 12 months of supplementation. Data are presented as mean \pm SEM.

\begin{tabular}{cccccccc}
\hline & \multicolumn{2}{c}{ Month 0 } & \multicolumn{2}{c}{ Month 12 } & \multicolumn{2}{c}{$\Delta$ Month 12-Month 0 } & \\
\cline { 2 - 6 } Fasting Serum Biomarkers & $\begin{array}{c}\text { Placebo } \\
(\boldsymbol{n}=\mathbf{6 5})\end{array}$ & $\begin{array}{c}\text { Resveratrol } \\
(\boldsymbol{n}=\mathbf{5 9 )}\end{array}$ & $\begin{array}{c}\text { Placebo } \\
(\boldsymbol{n}=\mathbf{6 5})\end{array}$ & $\begin{array}{c}\text { Resveratrol } \\
(\boldsymbol{n}=\mathbf{5 9 )}\end{array}$ & $\begin{array}{c}\text { Placebo } \\
(\boldsymbol{n}=\mathbf{6 5 )}\end{array}$ & $\begin{array}{c}\text { Resveratrol } \\
(\boldsymbol{n}=\mathbf{5 9 )}\end{array}$ & $\boldsymbol{P}$-Value \\
\hline Glucose (mmol/L) & $5.0 \pm 0.07$ & $5.0 \pm 0.07$ & $5.0 \pm 0.06$ & $5.0 \pm 0.06$ & $0.05 \pm 0.06$ & $-0.00 \pm 0.04$ & 0.499 \\
Insulin (mIU/L) & $7.2 \pm 0.46$ & $7.9 \pm 0.61$ & $8.0 \pm 0.42$ & $8.1 \pm 0.59$ & $0.82 \pm 0.42$ & $0.16 \pm 0.50$ & 0.308 \\
HOMA-IR & $1.6 \pm 0.12$ & $1.8 \pm 0.16$ & $1.8 \pm 0.11$ & $1.8 \pm 0.14$ & $0.20 \pm 010$ & $0.01 \pm 0.12$ & 0.236 \\
Triglycerides (mmol/L) & $1.1 \pm 0.06$ & $1.2 \pm 0.07$ & $1.2 \pm 0.06$ & $1.3 \pm 0.07$ & $0.08 \pm 0.04$ & $0.10 \pm 0.05$ & 0.800 \\
Total cholesterol (mmol/L) & $5.7 \pm 0.11$ & $5.6 \pm 0.18$ & $5.7 \pm 0.12$ & $5.6 \pm 0.18$ & $-0.03 \pm 0.07$ & $-0.01 \pm 0.08$ & 0.901 \\
LDL-cholesterol (mmol/L) & $3.7 \pm 0.11$ & $3.5 \pm 0.17$ & $3.6 \pm 0.11$ & $3.5 \pm 0.17$ & $-0.08 \pm 0.07$ & $-0.03 \pm 0.06$ & 0.561 \\
HDL-cholesterol (mmol/L) & $1.6 \pm 0.04$ & $1.6 \pm 0.05$ & $1.6 \pm 0.05$ & $1.6 \pm 0.05$ & $0.02 \pm 0.02$ & $-0.03 \pm 0.02$ & 0.168 \\
Hs-CRP (mg/L) & $2.0 \pm 0.24$ & $2.4 \pm 0.67$ & $2.2 \pm 0.43$ & $2.5 \pm 0.34$ & $0.22 \pm 0.43$ & $0.10 \pm 0.54$ & 0.862 \\
\hline
\end{tabular}

Abbreviations: HOMA-IR = Homeostatic Model Assessment of Insulin Resistance, LDL-cholesterol = low-density lipoprotein, $\mathrm{HDL}=$ high-density lipoprotein, Hs-CRP = high sensitivity C-reactive protein.

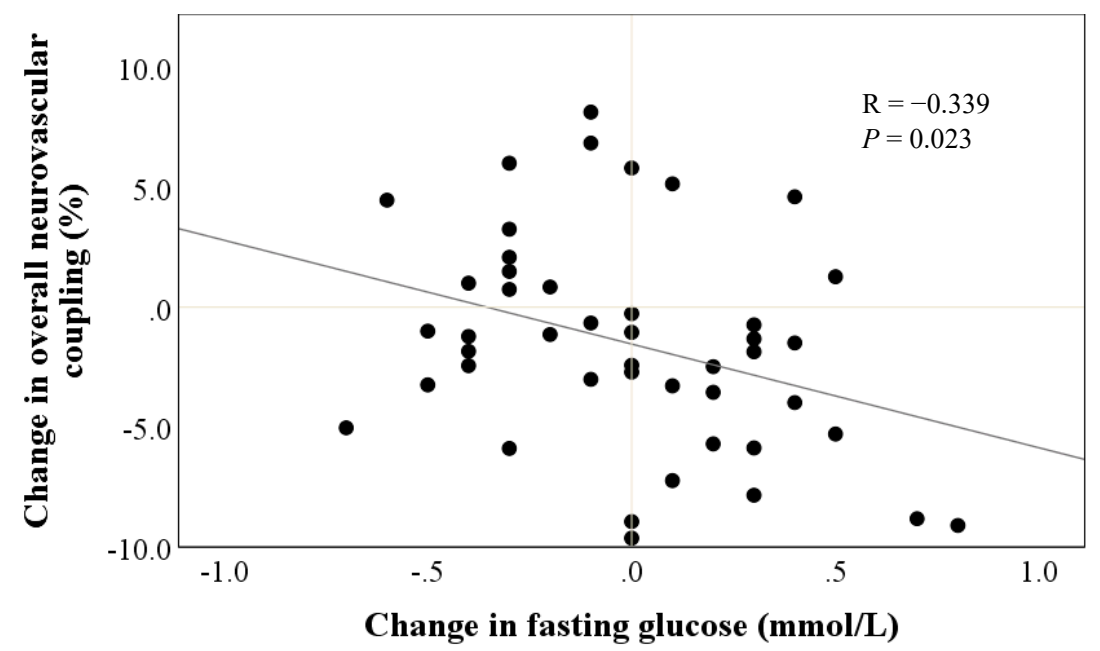

Figure 5. Association between treatment change in fasting glucose and treatment change in overall neurovascular coupling following resveratrol supplementation.

\subsection{Adverse Events}

A total of 12 adverse events were reported during the trial, four of which were serious conditions requiring hospitalisation, viz. a urinary tract infection, a bowel blockage, an oesophageal tear repair and a pre-scheduled operation on the lumbar spine. All four occurred in the placebo group. Of the remaining eight adverse events, four occurred in the resveratrol group but were not necessarily attributable to supplementation (viz. itching, menses, prolapsed bladder and a pre-scheduled left eye operation) and four occurred in the placebo group (viz. itching, exacerbation of gastric reflux, constipation and a pre-scheduled breast reduction procedure). Treatment compliance averaged $95 \%$ in both groups.

\section{Discussion}

In this 12-month trial, we sought to confirm the unique findings of our 14 week pilot study in postmenopausal women [16] and ascertain whether the benefits of low-dose resveratrol on cerebrovascular and cognitive functions could be sustained with long-term supplementation.

We observed an improvement in overall cognitive performance which appeared to be due to improvements in processing speed and cognitive flexibility. Both processing speed and cognitive flexibility are part of executive function that requires speed, perceptual reasoning and accuracy to accomplish tasks [24]. After peaking in the third decade of life, executive function declines at an estimated annual rate of -0.02 standard deviations [25]. In fact, slowing of processing speed and mental flexibility are among the first cognitive changes reported in healthy older adults [24]. 
This "slowing" can negatively affect performance on other neuropsychological tests such as verbal fluency [24]. Our observed improvement in overall cognitive performance with resveratrol $(d=0.18)$ could potentially reverse cognitive ageing by up to 10 years. Therefore, optimising executive function in healthy older adults may delay subsequent impairment across other cognitive domains.

Apart from our pilot study [16], there are only two clinical trials on cognitive effects of chronic resveratrol supplementation [12,26]. A six-month study by Witte et al. showed improvement of verbal memory in healthy older adults with a similarly low dose (200 mg/day) [12]. On the other hand, a study in young adults [26] reported a lack of interpretable cognitive effects following resveratrol supplementation (500 mg/day for a month), despite showing improvement of cerebrovascular function. This may be due to the high-performing cognitive status of this cohort, the shorter study duration or the higher dose of resveratrol given, bearing in mind that we have previously reported lower neurovascular coupling efficacy of resveratrol at higher doses [14].

We hypothesized that the observed cognitive benefits of resveratrol might be partly mediated by sustained improvement of endothelium-dependent vasodilator function, which modulates CBF during times of demand [11]. Supporting this, we found improved resting BFV, PI and attenuation of decline in neurovascular coupling following resveratrol supplementation. Allowing for normal ageing processes, cognitive decline and ultimately, dementia are linked to accelerated decline in resting CBF and CVR due to a decreased delivery of oxygen and nutrients in vulnerable brain regions such as the hippocampus [27]. In fact, a large population-based study in Rotterdam reported that healthy older adults with lower CBF measured by MRI performed significantly worse on tasks of information processing speed, executive and global cognitive function compared to those with higher CBF [28]. In addition, cerebral artery stiffness, marked by increased PI, is also associated with cognitive impairment [29] and predicts the progression from mild cognitive impairment to dementia [30]. Combination of low BFV and high PI can result in chronic hypoperfusion, which may cause progressive loss of neuronal function [31]. Given this evidence, our observation of improvements in resting BFV and PI with resveratrol highlights the ability of regular resveratrol supplementation to sustain cerebrovascular function, which may, in turn, preserve cognitive function in elderly women.

Evidence has shown that resveratrol can modulate CBF through multiple mechanisms including activation of Sirtuin-1, adenosine-monophosphate protein kinase and ER $\alpha$ and $\beta$ to increase endothelial NO synthase activity. This, in turn, increases NO production and bioavailability to facilitate vasodilator responsiveness and arterial smooth muscle relaxation during demand [11]. To assess the cerebral vasodilator response, hypercapnia provocation is commonly used to increase of blood $\mathrm{CO}_{2}$ concentration. The increase in BFV from resting values reflects dilation in the downstream microvasculature and thus is a good measure of global cerebral vasodilatation, independent of specific neuronal activation [32]. We did not observe enhancement of CVR to hypercapnia with resveratrol in this study, perhaps because cerebral vasodilator capacity was still optimal in our cohort of healthy elderly women. Older adults with established cardiovascular risk factors such as hypertension, dyslipidaemia or diabetes had lower CVR to hypercapnia compared to healthy older and young adults [32]. Moreover, impaired CVR to hypercapnia has been observed in individuals with mild cognitive impairment and Alzheimer's disease [33], implicating cerebral hypoperfusion in the pathogenesis of cognitive impairment.

Although we did not see any enhancement of CVR to hypercapnia, we found that resveratrol attenuated the decline of neurovascular coupling. Neurovascular coupling differs from CVR to hypercapnia as it represents localised changes in cerebral BFV in response to specific neuronal events. When neurons fire, the endothelium is activated to release NO, resulting in dilatation of local arterioles, which is detected as increased blood flow in the arteries supplying the activated brain region. Deficits in this synchronised action can lead to inadequate perfusion of critical brain regions, resulting in poor performance of cognitive tasks [34]. Therefore, impaired neurovascular coupling may be considered the beginning of a chain of events leading to a progressive decline in brain metabolism and cognition that characterizes dementia [35]. Identification of early cerebrovascular dysfunction has 
important diagnostic implications for future cognitive decline [36]. We have previously shown that poor cerebrovascular function is predictive of cognitive decline in healthy postmenopausal women [8].

We have previously shown that the beneficial effects of resveratrol on systemic vascular function are greater in those individuals with poorer vascular function at baseline [37]. This may account for the greater magnitude of improvement in neurovascular coupling seen in our pilot study of resveratrol supplementation in postmenopausal women [16] $(\mathrm{d}=0.71)$, which elicited a greater improvement in overall cognitive performance $(d=0.69)$ than observed in the present study, as the participants in the pilot study had much lower overall neurovascular coupling at baseline. In this study, we have once again found that lower cognitive function at baseline is associated with greater improvements in overall cognitive performance by resveratrol. Taken together, regular resveratrol supplementation may be more beneficial for improving cognitive function in older adults with higher level of baseline endothelial dysfunction or cognitive impairments. The present study not only confirms the significant benefits of resveratrol seen in the pilot study but, most importantly, it shows that these benefits are not short-lived but can be sustained with ongoing supplementation for at least 12 months. Indeed, data from the placebo-treated group revealed a further decline of neurovascular coupling over 12 months, even in healthy older women. The present study demonstrates the potential of resveratrol to attenuate this decline, thereby protecting higher brain function in the elderly.

We did not find any significant difference in systemic vascular function (BP, AC) or fasting glucose, insulin and lipids between the two treatments, although Timmers et al. had shown that resveratrol (150 mg/day for 30 days) can reduce plasma triglycerides and improve insulin sensitivity in obese male subjects (mean age: $52 \pm 2$ years, BMI: $31.6 \pm 0.7 \mathrm{~kg} / \mathrm{m}^{2}$ ) with low-grade chronic inflammation [38]. They also reported reduced inflammation; however, we did not observe an effect of resveratrol on hs-CRP, indicating that resveratrol is unlikely to alter cardiometabolic markers in elderly participants who are otherwise healthy (BMI: $25.6 \pm 0.3 \mathrm{~kg} / \mathrm{m}^{2}$ ) and without overt inflammation or other metabolic disturbances.

Interestingly, we found a significant inverse relationship between the supplementation-induced changes in fasting glucose and overall neurovascular coupling. Our finding is consistent with Witte et al., who observed a reduction in $\mathrm{HbA1C}$, the long-term biomarker of glucose control, which correlated with increased functional connectivity in the hippocampus assessed by functional MRI [12]. This is consistent with our previously published hypothesis that resveratrol might improve glucose uptake and/or reduce insulin demand by enhancing vasodilator function in skeletal muscle [39]. A recent meta-analysis has also shown that resveratrol can improve insulin sensitivity and insulin secretion in pancreatic $\beta$-cells and increase glucose uptake via Sirtuin- 1 or adenosine monophosphate protein kinase mediated pathways [40]. Resveratrol could also facilitate expression of glucose transporter type- 4 by acting on estrogen receptors on the endothelium and stimulate skeletal muscle glucose uptake [41], which could be an added metabolic benefit for postmenopausal women. Although we cannot rule out the exact mechanism of action, this finding could imply that resveratrol may induce improvements in energy metabolism through improvements of microcirculatory function, which could, in turn, protect neuronal function and counteract cognitive decline.

This is the first long-term study of effects of resveratrol supplementation in postmenopausal women. The 12-month duration not only increases confidence in the sustainability of any benefits but it also eliminates any influences of seasonality on the outcomes. Whilst generating further evidence on the benefits of resveratrol for healthy ageing, there are some obvious limitations to its application. Individuals with higher health consciousness and cognitive awareness tend to volunteer for such intervention trials, which may influence their compliance and performance and ultimately, the interpretation of the results. Moreover, as our sample included healthy postmenopausal women only, the results cannot be generalized to the entire population. Further trials are necessary to determine the extent of potential benefit for men and for specific risk groups, e.g., hypertensives and diabetics, of both sexes. 


\section{Conclusions}

Findings from this study confirm the results of our pilot study and demonstrate a sustained benefit of long-term low dose resveratrol supplementation, viz. the improvement of overall cognitive performance, which can be attributed, at least partly, to improvement of cerebral blood flow and vasodilator responsiveness during cognitive demands. We also demonstrated an association of resveratrol-induced changes in fasting glucose with improvements in endothelial vasodilator function, which warrants further investigation. The low dose of resveratrol was well tolerated over a period of 12 months without apparent side effects. Our findings support the adoption of resveratrol as a low-cost, effective intervention to help counteract the age and menopause-related accelerated cognitive decline in our ageing population. Subsequent publications from this study will report effects of resveratrol on bone health, physical function and quality of life measures which, collectively, will establish resveratrol as a viable intervention to promote healthy ageing in women.

Supplementary Materials: The following are available online at http://www.mdpi.com/2072-6643/12/3/828/s1, Table S1: Blood pressure (BP) and arterial compliance between resveratrol and placebo groups at baseline and after 12 months of supplementation. Data are presented as mean \pm SEM.

Author Contributions: R.H.X.W. and P.R.C.H. conceived the study design and supervised the intervention trial; J.J.T.Z. and R.H.X.W. undertook data collection and analysed the data; J.J.T.Z. drafted the manuscript under guidance from R.H.X.W. and P.R.C.H. All authors have read and agreed to the published version of the manuscript.

Funding: The study was supported by a National Health and Medical Research Council of Australia Dementia Research Fellowship awarded to Dr Rachel Wong (APP1106170). Evolva, Switzerland supplied the test materials and supplementary funding but had no role in the study design, data collection, analysis and interpretation or writing of the manuscript.

Acknowledgments: The authors thank Hamish Evans and Natasha Baker for their assistance with the study.

Conflicts of Interest: The authors declare no conflict of interest. The funders had no role in the design of the study; in the collection, analyses, or interpretation of data; in the writing of the manuscript, or in the decision to publish the results.

\section{References}

1. GBD 2016 Dementia Collaborators. Global, regional, and national burden of Alzheimer's disease and other dementias, 1990-2016: A systematic analysis for the Global Burden of Disease Study 2016. Lancet Neurol. 2019, 18, 88-106. [CrossRef]

2. Colditz, G.A.; Willett, W.C.; Stampfer, M.J.; Rosner, B.; Speizer, F.E.; Hennekens, C.H. Menopause and the risk of coronary heart disease in women. N. Engl. J. Med. 1987, 316, 1105-1110. [CrossRef] [PubMed]

3. Li, R.; Singh, M. Sex differences in cognitive impairment and Alzheimer's disease. Front. Neuroendocrinol. 2014, 35, 385-403. [CrossRef] [PubMed]

4. Taddei, S.; Virdis, A.; Ghiadoni, L.; Mattei, P.; Sudano, I.; Bernini, G.; Pinto, S.; Salvetti, A. Menopause is associated with endothelial dysfunction in women. Hypertension 1996, 28, 576-582. [CrossRef] [PubMed]

5. Matteis, M.; Troisi, E.; Monaldo, B.C.; Caltagirone, C.; Silvestrini, M. Age and sex differences in cerebral hemodynamics: A transcranial Doppler study. Stroke 1998, 29, 963-967. [CrossRef] [PubMed]

6. Arora, S.; Veves, A.; Caballaro, A.E.; Smakowski, P.; LoGerfo, F.W. Estrogen improves endothelial function. J. Vasc. Surg. 1998, 27, 1141-1147. [CrossRef]

7. Silvestrini, M.; Paolino, I.; Vernieri, F.; Pedone, C.; Baruffaldi, R.; Gobbi, B.; Cagnetti, C.; Provinciali, L.; Bartolini, M. Cerebral hemodynamics and cognitive performance in patients with asymptomatic carotid stenosis. Neurology 2009, 72, 1062-1068. [CrossRef] [PubMed]

8. Wong, R.H.; Evans, H.M.; Howe, P.R. Poor cerebrovascular function is an early marker of cognitive decline in healthy postmenopausal women. Alzheimer's Dement. Transl. Res. Clin. Interv. 2016, 2, 162-168. [CrossRef]

9. Weber, M.T.; Maki, P.M.; McDermott, M.P. Cognition and mood in perimenopause: A systematic review and meta-analysis. J. Steroid Biochem. Mol. Biol. 2014, 142, 90-98. [CrossRef]

10. Wong, R.; Howe, P.; Buckley, J.; Coates, A.; Kunz, I.; Berry, N. Acute resveratrol supplementation improves flow-mediated dilatation in overweight/obese individuals with mildly elevated blood pressure. Nutr. Metab. Cardiovasc. Dis. 2011, 21, 851-856. [CrossRef] 
11. Xia, N.; Förstermann, U.; Li, H. Resveratrol and endothelial nitric oxide. Molecules 2014, 19, $16102-16121$. [CrossRef] [PubMed]

12. Witte, A.V.; Kerti, L.; Margulies, D.S.; Flöel, A. Effects of resveratrol on memory performance, hippocampal functional connectivity, and glucose metabolism in healthy older adults. J. Neurosci. 2014, 34, 7862-7870. [CrossRef] [PubMed]

13. Kennedy, D.O.; Wightman, E.L.; Reay, J.L.; Lietz, G.; Okello, E.J.; Wilde, A.; Haskell, C.F. Effects of resveratrol on cerebral blood flow variables and cognitive performance in humans: A double-blind, placebo-controlled, crossover investigation. Am. J. Clin. Nutr. 2010, 91, 1590-1597. [CrossRef]

14. Wong, R.; Nealon, R.; Scholey, A.; Howe, P. Low dose resveratrol improves cerebrovascular function in type 2 diabetes mellitus. Nutr. Metab. Cardiovasc. Dis. 2016, 26, 393-399. [CrossRef]

15. Wong, R.; Raederstorff, D.; Howe, P. Acute resveratrol consumption improves neurovascular coupling capacity in adults with type 2 diabetes mellitus. Nutrients 2016, 8, 425. [CrossRef] [PubMed]

16. Evans, H.; Howe, P.; Wong, R. Effects of resveratrol on cognitive performance, mood and cerebrovascular function in post-menopausal women; a 14-week randomised placebo-controlled intervention trial. Nutrients 2017, 9, 27. [CrossRef]

17. Hsieh, S.; Schubert, S.; Hoon, C.; Mioshi, E.; Hodges, J.R. Validation of the Addenbrooke's Cognitive Examination III in frontotemporal dementia and Alzheimer's disease. Dement. Geriatr. Cogn. Disord. 2013, 36, 242-250. [CrossRef]

18. Altman, D.G.; Bland, J.M. Treatment allocation by minimisation. BMJ 2005, 330, 843. [CrossRef]

19. Wagshul, M.E.; Eide, P.K.; Madsen, J.R. The pulsating brain: A review of experimental and clinical studies of intracranial pulsatility. Fluids Barriers CNS 2011, 8, 5. [CrossRef]

20. Gershon, R.C.; Wagster, M.V.; Hendrie, H.C.; Fox, N.A.; Cook, K.F.; Nowinski, C.J. NIH toolbox for assessment of neurological and behavioral function. Neurology 2013, 80, S2-S6. [CrossRef]

21. Cohen, J. Statistical power analysis. Curr. Dir. Psychol. Sci. 1992, 1, 98-101. [CrossRef]

22. Benjamini, Y.; Hochberg, Y. Controlling the false discovery rate: A practical and powerful approach to multiple testing. J. R. Stat. Soc. Ser. B 1995, 57, 289-300. [CrossRef]

23. Moher, D.; Hopewell, S.; Schulz, K.F.; Montori, V.; Gotzsche, P.C.; Devereaux, P.J.; Elbourne, D.; Egger, M.; Altman, D.G. CONSORT 2010 statement: Updated guidelines for reporting parallel group randomised trials. BMJ (Clin. Res. Ed) 2010, 340, c332. [CrossRef]

24. Harada, C.N.; Love, M.C.N.; Triebel, K.L. Normal cognitive aging. Clin. Geriatr. Med. 2013, 29, 737-752. [CrossRef] [PubMed]

25. Salthouse, T. Consequences of age-related cognitive declines. Annu. Rev. Psychol. 2012, 63, 201-226. [CrossRef] [PubMed]

26. Wightman, E.L.; Haskell-Ramsay, C.F.; Reay, J.L.; Williamson, G.; Dew, T.; Zhang, W.; Kennedy, D.O. The effects of chronic trans-resveratrol supplementation on aspects of cognitive function, mood, sleep, health and cerebral blood flow in healthy, young humans. Br. J. Nutr. 2015, 114, 1427-1437. [CrossRef] [PubMed]

27. Ruitenberg, A.; den Heijer, T.; Bakker, S.L.; van Swieten, J.C.; Koudstaal, P.J.; Hofman, A.; Breteler, M.M. Cerebral hypoperfusion and clinical onset of dementia: The Rotterdam Study. Ann. Neurol. Off. J. Am. Neurol. Assoc. Child Neurol. Soc. 2005, 57, 789-794. [CrossRef] [PubMed]

28. Poels, M.M.; Ikram, M.A.; Vernooij, M.W.; Krestin, G.P.; Hofman, A.; Messen, W.J.; Van der Lugt, A.; Breteler, M.M. Total cerebral blood flow in relation to cognitive function: The Rotterdam Scan Study. J. Cereb. Blood Flow Metab. 2008, 28, 1652-1655. [CrossRef] [PubMed]

29. Harris, S.; Reyhan, T.; Ramli, Y.; Prihartono, J.; Kurniawan, M. Middle cerebral artery pulsatility index as predictor of cognitive impairment in hypertensive patients. Front. Neurol. 2018, 9, 538. [CrossRef]

30. Chung, C.-P.; Lee, H.-Y.; Lin, P.-C.; Wang, P.-N. Cerebral artery pulsatility is associated with cognitive impairment and predicts dementia in individuals with subjective memory decline or mild cognitive impairment. J. Alzheimer's Dis. 2017, 60, 625-632. [CrossRef]

31. Trudeau, F.; Gagnon, S.; Massicotte, G. Hippocampal synaptic plasticity and glutamate receptor regulation: Influences of diabetes mellitus. Eur. J. Pharm. 2004, 490, 177-186. [CrossRef]

32. Gröschel, K.; Terborg, C.; Schnaudigel, S.; Ringer, T.; Riecker, A.; Witte, O.W.; Kastrup, A. Effects of physiological aging and cerebrovascular risk factors on the hemodynamic response to brain activation: A functional transcranial Doppler study. Eur. J. Neurol. 2007, 14, 125-131. [CrossRef] [PubMed] 
33. Richiardi, J.; Monsch, A.U.; Haas, T.; Barkhof, F.; Van de Ville, D.; Radü, E.W.; Kressig, R.W.; Haller, S. Altered cerebrovascular reactivity velocity in mild cognitive impairment and Alzheimer's disease. Neurobiol. Aging 2015, 36, 33-41. [CrossRef] [PubMed]

34. Jennings, J.R.; Muldoon, M.F.; Ryan, C.; Price, J.C.; Greer, P.; Sutton-Tyrrell, K.; Van Der Veen, F.M.; Meltzer, C.C. Reduced cerebral blood flow response and compensation among patients with untreated hypertension. Neurology 2005, 64, 1358-1365. [CrossRef] [PubMed]

35. De la Torre, J.; Stefano, G. Evidence that Alzheimer's disease is a microvascular disorder: The role of constitutive nitric oxide. Brain Res. Rev. 2000, 34, 119-136. [CrossRef]

36. Iadecola, C. Neurovascular regulation in the normal brain and in Alzheimer's disease. Nat. Rev. Neurosci. 2004, 5, 347. [CrossRef] [PubMed]

37. Wong, R.H.; Berry, N.M.; Coates, A.M.; Buckley, J.D.; Bryan, J.; Kunz, I.; Howe, P.R. Chronic resveratrol consumption improves brachial flow-mediated dilatation in healthy obese adults. J. Hypertens. 2013, 31, 1819-1827. [CrossRef]

38. Timmers, S.; Konings, E.; Bilet, L.; Houtkooper, R.H.; van de Weijer, T.; Goossens, G.H.; Hoeks, J.; van der Krieken, S.; Ryu, D.; Kersten, S.; et al. Calorie restriction-like effects of 30 days of resveratrol supplementation on energy metabolism and metabolic profile in obese humans. Cell Metab. 2011, 14, 612-622. [CrossRef]

39. Wong, R.H.; Howe, P.R. Resveratrol Counteracts Insulin Resistance-Potential Role of the Circulation. Nutrients 2018, 10, 1160. [CrossRef]

40. Zhu, X.; Wu, C.; Qiu, S.; Yuan, X.; Li, L. Effects of resveratrol on glucose control and insulin sensitivity in subjects with type 2 diabetes: Systematic review and meta-analysis. Nutr. Metab. 2017, 14, 60. [CrossRef]

41. Deng, J.-Y.; Hsieh, P.-S.; Huang, J.-P.; Lu, L.-S.; Hung, L.-M. Activation of estrogen receptor is crucial for resveratrol-stimulating muscular glucose uptake via both insulin-dependent and-independent pathways. Diabetes 2008, 57, 1814-1823. [CrossRef] [PubMed]

(C) 2020 by the authors. Licensee MDPI, Basel, Switzerland. This article is an open access article distributed under the terms and conditions of the Creative Commons Attribution (CC BY) license (http://creativecommons.org/licenses/by/4.0/). 